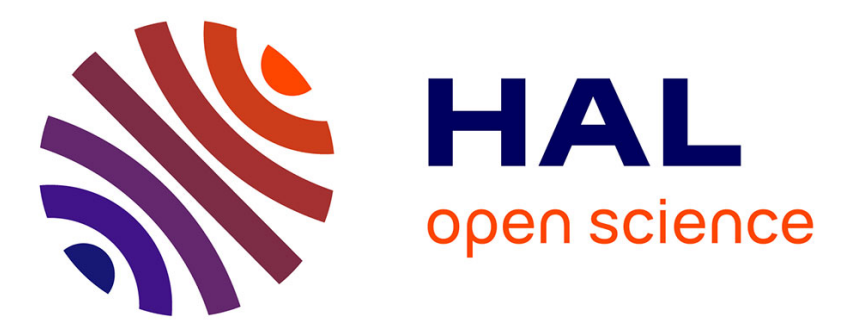

\title{
A Conventional Dialogue Model based on Empirically Specified Dialogue Games
}

Guillaume Dubuisson Duplessis, Alexandre Pauchet, Nathalie Chaignaud, J.-Ph Kotowicz

\section{- To cite this version:}

Guillaume Dubuisson Duplessis, Alexandre Pauchet, Nathalie Chaignaud, J.-Ph Kotowicz. A Conventional Dialogue Model based on Empirically Specified Dialogue Games. IEEE 27th International Conference on Tools with Artificial Intelligence, Nov 2015, Vietri sul Mare, Italy. pp.997-1004, 10.1109/ICTAI.2015.143 . hal-01228571

\section{HAL Id: hal-01228571 \\ https://hal.science/hal-01228571}

Submitted on 13 Nov 2015

HAL is a multi-disciplinary open access archive for the deposit and dissemination of scientific research documents, whether they are published or not. The documents may come from teaching and research institutions in France or abroad, or from public or private research centers.
L'archive ouverte pluridisciplinaire HAL, est destinée au dépôt et à la diffusion de documents scientifiques de niveau recherche, publiés ou non, émanant des établissements d'enseignement et de recherche français ou étrangers, des laboratoires publics ou privés. 


\section{A Conventional Dialogue Model based on Empirically Specified Dialogue Games}

\author{
Guillaume Dubuisson Duplessis \\ LITIS - INSA Rouen, Rouen, France \\ LIMSI-CNRS, Paris, France \\ gdubuisson@limsi.fr
}

\author{
Alexandre Pauchet \\ LITIS - INSA Rouen \\ Rouen, France \\ pauchet@insa-rouen.fr
}

\author{
Nathalie Chaignaud \\ LITIS - INSA Rouen \\ Rouen, France \\ chaignaud@insa-rouen.fr j.kotowicz@insa.ueuromed.org
}

\begin{abstract}
Our work aims at designing a dialogue manager dedicated to agents that interact with humans. In this article, we show how empirically specified dialogue games can be employed on both interpretative and generative levels of dialogue management. We present Dogma, an open-source module that can be used by an agent to manage its conventional communicative behaviour. We show that our library of dialogue games can be used into Dogma to generate fragments of dialogue that are strongly coherent from a human perspective.
\end{abstract}

Keywords-Human-Machine Interaction; Dialogue Management; Dialogue Games;

\section{INTRODUCTION}

The range of artificial interactive agents is wide: embodied conversational agents [1], mixed-initiative assisting agents [2], and so on [3]. When designing an interactive agent, dialogue management is a key feature [2], [4], [5] since dialogue is an efficient and natural way of communicating with humans. However, designing the communication model of an interactive agent is challenging [6] and often leads to the integration of rigid dialogue management processes, such as a keyword spotter.

Dialogue is a joint and opportunistic activity [7]: the interlocutors coordinate their contributions to co-construct and co-control the dialogue. To model dialogue with an overall coherence, high-level structures have to be considered (e.g., formalising utterances according to their semantic content). One striking observation in human dialogue is the presence of recurrent patterns. They correspond to sequences of utterances that are frequently reoccurring (e.g., a question/answer pair) [8], [9]. We share the point of view of [10] that recurrent dialogue patterns occurring in Human-Human $(\mathrm{H}-$ $\mathrm{H})$ interaction can be exploited to model Human-Machine (H-M) interaction.

In previous work [11], we have presented our long-term goal to build a mixed-initiative assistant for information retrieval for the CISMeF system [12]. To this end, we have also described a data-driven methodology dedicated to the extraction of dialogue patterns, as well as its implementation from corpus collection to the formalisation of dialogue games [13] from dialogue patterns. This paper describes

\footnotetext{
* This work was partly supported by the NARECA project (ANR-13CORD-0015).
}

the next step, i.e. how an interactive agent can employ dialogue games to manage its conventional communicative behaviour. These dialogue games constitute the basic interaction units manipulated by the deliberative process of an interactive agent. We point out how these structures can be fruitfully exploited during the dialogue management process. We describe our open-source software DoGMA, designed as a module included in a dialogue manager. Finally, we present promising results from the first version of our system. We show how empirically specified dialogue games implemented into DoGMA generate fragments of interaction that are strongly coherent from a human perspective.

Section II draws some links with related work, with a focus on dialogue patterns and dialogue management. Section III presents a brief description of our data-driven methodology. Section IV describes our dialogue game framework used to formalise interaction patterns. Section V shows how dialogue games can concretely and fruitfully be used in the dialogue management process of an interactive agent. We present DogmA, the dialogue game manager we propose, that implements our empirically specified dialogue games. Section VI deals with the evaluation of the dialogue games in terms of coherence, and discusses the contribution of the model. Lastly, section VII concludes this article.

\section{RELATED WORK}

Plan-based approaches view interaction patterns as an evidence of a plan followed by dialogue participants (DP), and focus on the intentional structure of dialogue [14]. The idea is that DPs infer the underlying plan behind an utterance to produce a cooperative response (see, e.g., TRAINS [15] or Collagen [16], [17]). On the other hand, conventional approaches consider patterns as conventional devices used by DPs. This has lead to models describing admissible sequences of utterances without a focus on the underlying intentions (e.g., dialogue grammars [18]).

These two approaches are often viewed as opposite, although some researchers argue that they are complementary [9], [19], [20]: communication processes are joint actions between DPs that require coordination of participatory actions [7]. Nevertheless, DPs cannot deliberate indefinitely and coordination must stand on elements such as conventions reflected by interaction patterns. Thus, dialogue can be 
considered as a shared and dynamic activity that requires both high-level deliberative reasoning processes and lowlevel reactive responses.

In this article, we consider dialogue games used to explain human dialogue and to generate artificial dialogues dedicated to humans [13]. Their purpose is to capture the conventions of human interaction. To the best of our knowledge, dialogue games have received only few attention from practical applications in the human-computer interaction field. On a theoretical level, dialogue games have been seen as initiativeresponse units [9], and as structures capturing commitments created during dialogue [19]. On both practical and formal level, rules of dialogue games have been represented as recursive transition networks [21], [22]. As [9], [19], we propose to use a hybrid reactive/deliberative architecture where a theory of joint actions may serve as a "semantics" to the interaction patterns described as dialogue games.

\section{DATA-Driven Methodology}

Our work is based on a data-driven methodology similar to [10]. It is a generalisation of the classic approach used to study corpora in various research fields such as linguistic psychology, and can be broken down into a sequence of steps: collection of a $\mathrm{H}-\mathrm{H}$ corpus, annotation of interaction units, pattern extraction and modelling. This methodology aims to extract dialogue patterns in order to enrich highlevel structures, useful for the dialogue management process of an agent interacting with a human.

Our long-term goal is to build a mixed-initiative assistant for information retrieval for the CISMEF system [12]. To that end, we presented an implementation of our methodology from corpus collection to pattern extraction in [11]. This application took place on a task-oriented corpus involving collaborative information search between an expert and a novice user, performed on a medical search engine. The annotation step was performed with the DIT++ annotation scheme of communicative functions [23]. The extraction process was carried out on $2 / 3$ of our initial corpus (the other third constitutes the reference corpus used in our validation). All in all, we formed a database of task-oriented dialogue games available to an interactive agent. It contains 8 items: 3 action-oriented dialogue games and 5 informationseeking-oriented dialogue games ${ }^{1}$. They mainly formalize initiative-response patterns consisting in adjacency pairs with preferred and dis-preferred second pair parts [24] (see, e.g., turns 8 and 9 in table II). We have shown that this database provides a suitable coverage of $\mathrm{H}-\mathrm{H}$ interaction patterns appearing in the reference corpus [11].

\footnotetext{
${ }^{1}$ Action-oriented game types are: Offer, Request, Suggestion. Information-seeking game types are: OpenInterrogation, ChoiceGame, VerificationGame, NegativeVerificationGame, YNInterrogationGame.
}

\section{Dialogue Game Framework}

\section{A. Model}

We consider recurrent dialogue patterns as conventional devices used by dialogue participants to coordinate their communicative actions in dialogue seen as a shared, dynamic and opportunistic activity. From our perspective, conventions can fruitfully be captured by a dialogue game structure to improve the conventional communicative capabilities of agents interacting with a human. A dialogue game is a conventional bounded joint activity between an initiator and a partner. Rules of the dialogue game specify the expected moves $^{2}$ for each DP. Participants are expected to play their roles by making moves according to the current stage of the game. This activity is temporarily activated during the dialogue for a specific goal (e.g., information-seeking, action-seeking).

A detailed account of our formalisation of the dialogue game structure can be found in [11]. We provide here an overview of our model, sufficient for the purposes of this article. A dialogue game is defined by a type and a subject. For instance, "Request(addKeyword(heart))" represents a dialogue game of type "Request" with subject "addKeyword(heart)" (which is the formalisation of the action "add the keyword "heart' to the query", further described in section VI-A1). A dialogue game is a bilateral and shared structure which defines for the initiator of the game and for the partner: (i) entry conditions (which must hold before entering the game), (ii) exit conditions (which define the success or failure status of the game), (iii) rules (which define the expectations/obligations of dialogue participants in the game), and (iv) the effects of dialogue moves in the context of this game (see, e.g., table I).

These aspects of dialogue games are modelled through the notions of social commitment and commitment store, inspired by dialectical systems [19], [26]. Social commitments are commitments that bind a speaker to a community [27]. They are public (unlike mental states such as belief, desire, intention), and are stored in a commitment store. Our formalisation classically distinguishes propositional commitments from action commitments. Propositional commitments concern those that do not deal with future action such as when $x$ says "Charlie is well-known.". On the other hand, action commitments concern those dealing with future action such as "I will make you a drawing tonight.". Thus, they represent actions that the agent is committed to perform in the future. Besides, action commitments may be either dialogical (they are valid in the context of a game) or extra-dialogical (their validity is not subject to a game). Social commitments are stored in a certain state. States can be distinguished between the default state (inactive,

\footnotetext{
${ }^{2}$ Moves correspond to a context-change approach to dialogue acts [23], [25]. They take the form: $f(s, c)$ where $f$ is the communicative function, $s$ the speaker that produces this act and $c$ the semantic content.
} 
Ina), the state in which the commitment is active (created, Crt) and various states of inactivity subsequent to a period of activation (cancelled (Cnl), fulfilled (Ful) and failed (Fal)). The state of social commitments evolves through operations applied on the commitment store (e.g., creation, cancellation, satisfaction). Commitments are formalised as 4-arity predicates: $\mathrm{C}(x, y, c, s)$ (meaning that commitment " $x$ is committed towards $y$ about content $c$ " is in state $s)$.

The content of action commitments can be combined in several ways including (but not limited to) the alternative $(\alpha \mid \beta)$ and the conditional statement $(\alpha \Rightarrow \beta)$ ( $\beta$ will occur if $\alpha$ does). Action commitments in our model make it possible to represent two high-level kinds of commitments: expectations/obligations and production rules. Expectations/obligations are represented with firstorder commitments. For instance, when enclosed in a dialogical action commitment contracted by $y$, the content "acceptRequest $(y, \alpha) \mid \operatorname{declineRequest}(y, \alpha)$ " means that dialogue participant $y$ is expected to produce either an "acceptRequest" move, or a "declineRequest" move about action $\alpha$. Production rules encode conventional sequences of dialogue moves. They are represented with secondorder commitments. For example, when enclosed in a dialogical action commitment contracted by $y$, the production rule "request $(x, \alpha) \Rightarrow \mathrm{C}_{\mathrm{g}}(y$,acceptRequest $(y, \alpha) \mid$ declineRequest $(y, \alpha), \mathbf{C r t})$ "3 specifies that the occurrence of the dialogue move "request $(x, \alpha)$ " commits dialogue participant $y$ to the production of an acceptance or a declination (by the activation of a first-order commitment).

All in all, the commitment store represents public commitments contracted by DPs at a given time of the interaction. These commitments represent positions about propositions, promises on the (non-)fulfilment of actions such as dialogue moves, and production rules encoding conventional sequences of dialogue moves. Furthermore, the commitment store contains a representation of dialogue games that are being suggested, currently being played, and closed.

\section{B. Example: the Request Game}

The definition of the "Request" dialogue game is presented in table I. This game allows the initiator to request the carrying out of an action by the partner. Entry conditions specify that the partner must not be already committed on the requested action $\alpha$, i.e. either on its occurrence or its non-occurrence. The success conditions are the same for the two participants: the game is a success once the partner is committed to perform action $\alpha$. Similarly, the failure conditions state that the game is a failure if the attempt to commit the partner on $\alpha$ fails (i.e. the partner refuses to contract an active commitment about $\alpha$ ). Rules specify that the initiator of the game is committed to play a "request"

\footnotetext{
${ }^{3} g$ refers to the context of this dialogical commitment.
}

dialogue move, and that the partner is committed to play an "acceptRequest" or a "declineRequest" move if $x$ plays a "request" move. Finally, effects precise that, in the context of this game, playing an "acceptRequest" or a "declineRequest" move for the partner commits him to perform $\alpha$, or to the failure of the committing attempt about $\alpha$.

Table II presents an example of dialogue involving this dialogue game (formal descriptions of other involved dialogue games such as "ChoiceGame" can be found in [11]). Utterances 11 and 12 constitute the body of a "Request" dialogue game about the subject "addKeyword(heart)", initiated by interlocutor $y$ with partner $x$. This adjacency pair emerges from the conformance to the rules of the dialogue game. Interlocutor $y$ plays a "request" move in utterance 11 in the context of the "Request" dialogue game. The partner $x$ is thus committed to play either an acceptance or a declination (as shown in the "Expected acts" column). Interlocutor $x$ chooses to play an "acceptRequest" move in utterance 12, thus committing himself to perform the action "addKeyword(heart)". Hence, success conditions of this game are reached.

\section{The Contextualisation Game}

In addition to dialogue games, our model includes communication games that are dedicated to more general interaction processes (e.g., mutual understanding or turn-taking). These games are simpler in the sense that they are always activated. Hence, their structure comes down to a set of rules expressed as dialogical action commitments.

Dialogue is a joint and opportunistic activity [7] in which DPs coordinate their contributions to co-construct and cocontrol the dialogue. Dialogue games are managed by DPs in a process that presents three typical phases: the entry, the body and the exit of the joint activity. Establishment and closing of dialogue games have been described by a negotiation metaphor [28]. Our model includes the communication game of contextualisation which implements a version of this process that may involve two proposition phases (one for the entry and one for the exit). Dialogue example in table II presents two explicit entry phases: one for a choice dialogue game initiated by $x$ (utterances $1-2$ ) and another for an open interrogation dialogue game initiated by $y$ (utterances 6-7). Entry phases follow a common structure showing a proposition to enter the game (via a "prop.in" move) followed by an acceptance (via an "acc.in" move). Structure of an explicit exit phase is similar to the one of an entry phase. Exit phases can be realised implicitly via the reaching of success or failure conditions of the dialogue game.

\section{Dialogue Games and Dialogue Management}

\section{A. Interpretative and Generative Aspects of our Model}

$\mathrm{H}-\mathrm{M}$ dialogue models inspired by dialectical ones present a strong interest for modelling H-M interaction [20]. Their 
Table I

The Request Dialogue Game (EXPLAined in SECTION IV-B).

\begin{tabular}{|l|l|l|}
\hline$g=$ Request $(\alpha)$ & Initiator $(x)$ & Partner $(y)$ \\
\hline \hline Entry & & $\mathrm{C}(y, \alpha$, Ina $)$ and $\mathrm{C}(y, \neg \alpha$, Ina $)$ \\
Success & $\mathrm{C}(y, \alpha, \mathbf{C r t})$ & $\mathrm{C}(y, \alpha, \mathbf{C r t})$ \\
Failure & $\mathrm{C}(y, \alpha, \mathbf{F a l})$ & $\mathrm{C}(y, \alpha$, Fal $)$ \\
\hline Rules & request $(x, \alpha)$ & request $(x, \alpha) \Rightarrow \mathrm{C}_{\mathrm{g}}(y$, acceptRequest $(y, \alpha) \mid \operatorname{declineRequest}(y, \alpha)$, Crt $)$ \\
\hline Effects & & acceptRequest $(y, \alpha) \Rightarrow \mathrm{C}(y, \alpha$, Crt $), \operatorname{declineRequest}(y, \alpha) \Rightarrow \mathrm{C}(y, \alpha$, Fal $)$ \\
\hline
\end{tabular}

Table II

EXCERPT FROM A QUERY BUILDING DIALOGUE ON A MEDICAL SEARCH ENGINE BETWEEN TWO INTERLOCUTORS $(x$ AND $y)$ INVOLVING SEVERAL DiALOGUE GAMES (SEE SECTIONS IV-B AND V-C). THE "MOVE" COLUMN SHOWS THE COMMUNICATIVE FUnCTION BEING PLAYED. THE "GAME" COLUMN PRESENTS THE GAME IN WHICH THE MOVE OCCURS. THE "EXPECTED MOVES" COLUMN DESCRIBES THE COMMUNICATIVE FUNCTIONS OF THE MOVES THAT ARE EXPECTED ACCORDING TO THE COMMITMENT STORE (“<” IS THE PRIORITY RELATION, “|” REPRESENTS AN ALTERNATIVE),

\begin{tabular}{|c|c|c|c|c|}
\hline & Move & Game & Utterance & Expected moves \\
\hline 1 & prop.in & Contextualisation & $x$ : May I suggest some keywords? & $y:$ acc.in $\mid$ ref.in \\
\hline 2 & acc.in & - & $y:$ Yes. & $x$ : choiceQuestion \\
\hline 3 & choiceQuestion & ChoiceGame & $\begin{array}{l}x: \text { Which keyword would you choose among: 'heart', } \\
\text { 'myocardium', 'infarction', 'angiocardiography'? }\end{array}$ & $y:$ answer | ignore \\
\hline 4 & answer & - & $y$ : Well, not 'infarction'. & $\begin{array}{l}y \text { : answer } 1 \text { ignore } \\
\end{array}$ \\
\hline 5 & answer & - & ... not 'angiocardiography'. & $y$ : answer | ignore \\
\hline 6 & prop.in & Contextualisation & Can I ask you something? & $x$ : acc.in $\mid$ ref.in $<y$ : answer $\mid$ ignore \\
\hline 7 & acc.in & - & $x:$ Sure! & $y$ : setQuestion $<y$ : answer $\mid$ ignore \\
\hline 8 & setQuestion & OpenInterrogation & $y$ : What is the definition of the term 'myocardium'? & \begin{tabular}{l|l}
$x:$ answer & ignore $<$ \\
$y:$ answer & ignore
\end{tabular} \\
\hline 9 & answer & - & $\begin{array}{l}x: \text { A definition of 'myocardium' is: 'The muscle tissue of } \\
\text { the heart. It is composed of [...]' }\end{array}$ & $y$ : answer $\mid$ ignore \\
\hline $\begin{array}{l}10 \\
11\end{array}$ & $\begin{array}{l}\text { answer } \\
\text { request }\end{array}$ & $\begin{array}{l}\text { ChoiceGame } \\
\text { Request }\end{array}$ & $\begin{array}{l}y \text { : Then, I would choose keyword 'heart'! } \\
\text { Please, add this term to the query. }\end{array}$ & $\mathrm{x}$ : acceptRequest $\mid$ declineRequest \\
\hline 12 & acceptRequest & - & $x:$ All right! & \\
\hline
\end{tabular}

main advantage lies in their normative nature in that they constrain the communicative behaviour of the DPs. From a computational perspective, these models have two important interests for an interactive agent. They present an interpretative interest that makes it possible to determine the legality of a dialogue move produced by a DP. Therefore the agent can adapt its reaction according to whether a dialogue move is legal or not. Besides, this kind of model also presents a generative interest: it makes it possible to assist the system for the production of a response by limiting its attention to the legal moves that are expected from the agent.

The normative aspect of our model can be found in the idea of dialogue games as structures capturing social commitments (i.e. that encode conventions of human interaction). In the course of interaction, DPs gradually contract social commitments that not only constrain the communicative behaviour of the system, but that of the human participant as well.

From an interpretative point of view, our model considers the legality of a dialogue move from different perspectives. A dialogue move can be forbidden or expected depending on contracted social commitments. Among a list of expected dialogue moves, a particular one can have priority based on the partial ordering of social commitments (legality and priority are exemplified in section $\mathrm{V}-\mathrm{C}$ ). The interpretative role of a dialogue manager based on dialogue games is to classify a dialogue move according to these criteria, and react accordingly. This is exemplified by the generic algorithm V.1. The dialogue move that just occurs is first assessed against the contracted social commitments (line 1). Then, it is processed according to its legality status: a legal dialogue move (i.e. an allowed, expected and priority move) leads to a standard update of the dialogue context (line 6). Other cases (i.e. forbidden, non-priority or unexpected move) involve a case-specific dialogue policy (lines $3,8,11)$. From our perspective, these policies depend on the interactive agent that is being designed. For instance, forbidden dialogue moves can simply be prohibited in debate situations [29].

From a generative point of view, our model makes it possible to compute the conventionally expected dialogue moves from the system by examining its contracted dialogical commitments. It provides conventional reasons to the production of dialogue moves by taking into account the state of the commitment store as well as the activated dialogue games. These elements should be considered during the deliberative process of the interactive agent.

\section{B. Implementation: Dogma Module}

DoGmA (for "DialOgue Game MAnager") is a dialogue game-based normative module that manages conventional 

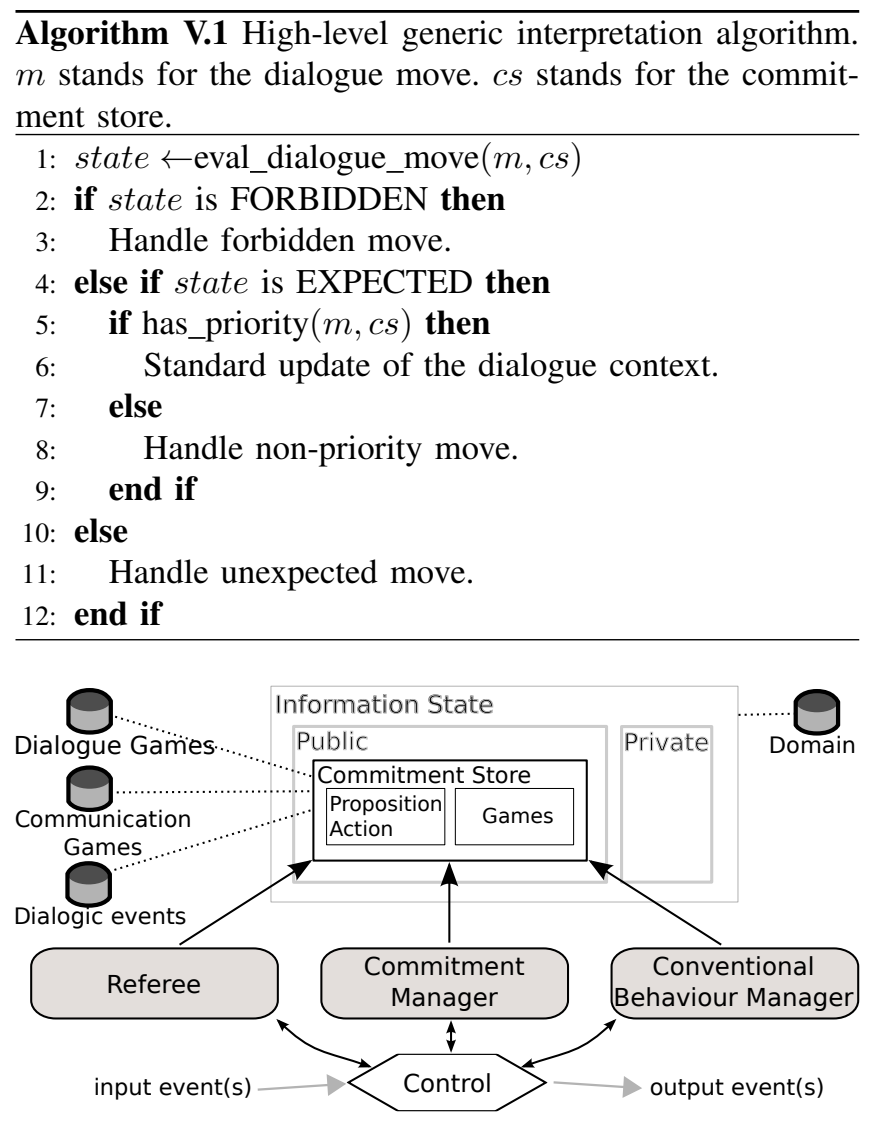

Figure 1. Dogma Architecture

interaction in a two-interlocutor dialogue. It is designed as a part of a dialogue manager of an interaction system. DoGMA adopts the information state-based approach to dialogue management [25]. It is in charge of the update and the exploitation of the commitment store located in the information state of the dialogue manager.

Figure 1 presents the architecture of Dogma. The system is centred around the information state, classically divided into two parts: the public part that includes the commitment store, and the private part. The commitment store is the main component of DogMA. It consists of a partially ordered set of social commitments contracted by the speakers. To be more specific, it contains for each DP the public commitments in terms of propositions (as extradialogical commitments) and actions (as extra-dialogical and dialogical commitments). Besides, it contains a representation of dialogue games that are being suggested, currently being played, and closed. The commitment manager has a $\mathrm{read} / \mathrm{write}$ access to the commitment store. Its role is to keep the commitment store up to date after the occurrence of events such as dialogue moves. DoGMA provides two components with a read access to the commitment store: (i) the referee that evaluates the legality of a dialogue move (cf. algorithm V.1), and (ii) the conventional behaviour manager that identifies the conventionally expected dialogue moves from each DP. The referee and commitment manager components are solicited during the interpretative phase of the dialogue manager control algorithm. The conventional behaviour manager is mainly called during the generative phase of the control algorithm.

Dogma is configured by three main resources: (i) the dialogic events library, (ii) the communication games library, and the (iii) dialogue games library. These resources define the dialogue moves and games that are available during the interaction. Last but not least, the domain library brings the domain-dependent elements of the application, namely: (i) the semantics of the dialogue move content, and (ii) a specification of the allowed combinations between dialogue games.

We have developed Dogma using the Scala programming language ${ }^{4}$. The source code is freely available under the GPLv3 licence ${ }^{5}$, and can currently be found at https://labanquise.insarouen.fr/projects/dogma/.

\section{Example of usage of DogmA}

Table II presents an excerpt of a query building dialogue on a medical search engine between two DPs. It shows how a dialogue manager can take advantage of DogmA to manage the conventional part of the interaction. From utterance 1 to 10 is shown the playing of a choice dialogue game initiated by $x$ (in utt. 1-2) that is interrupted by an open interrogation game initiated by $y$ (in utt. 6-9), and followed by a request game previously described in section IV-B. First, this example shows that the body of a dialogue game is not restricted to a pair of moves but can span several ones (e.g., utt. 3, 4, 5 and 10). Next, Dogma allows the combination of dialogue games such as embedding (cf. utt. 6-9). A consequence of such a combination happens on the priority of social commitments representing move expectations: moves expected in the embedded game take precedence over those of the parent game. For instance, an answer to the open interrogation game has priority over moves of the choice game after utt. 8, due to conversational precedence (the same occurs after utt. 6 and 7). Eventually, this example exposes the expected moves derived from the commitment store in the last column of the table. These expected moves are computed by the conventional behaviour manager which provides legal moves that are conventionally expected from DPs given the current dialogue state. As such, they represent both conventional expectations from the other DP and conventional reasons to the production of given moves. For example, after utt. 6, interlocutor $y$ conventionally expects an acceptation or declination about the establishment of the open interrogation game, and still has

\footnotetext{
${ }^{4}$ See http: //www.scala-lang.org/.

${ }^{5}$ See http://www.gnu.org/copyleft/gpl.html.
} 
conventional motivations to respond to the choice question asked by $x$ in utt. 3 .

\section{Evaluation}

Our goal is to evaluate the ability of our system to generate short fragments of interaction that are meaningful to humans (while being combinable by a dialogue planner). We adopted a validation process as a test "a la Turing". It consists in comparing a set of $\mathrm{H}-\mathrm{H}$ interaction patterns observed in our corpus to a set of interaction patterns automatically generated by our system. This variant involves experts whose task is to analyse a sample of interaction patterns (mixing real ones and generated ones) in order to determine if the two sets can easily be distinguished. This experiment leads us to the creation of four databases of dialogue patterns: (i) three databases of automatically generated interaction patterns by three different processes, and (ii) a database of interaction patterns from $\mathrm{H}-\mathrm{H}$ interactions.

\section{A. Databases of Interaction Patterns}

\section{1) Databases of Generated Patterns:}

Semantic Representation: We first manually established a semantic database of questions, propositions and actions occurring in the dialogue patterns of the study corpus. Our formalisation is a reduced semantic representation with a domain-dependent level of granularity similar to the one proposed by [30]. It is based on predicate logic without quantification. The use of such a simple semantic representation for practical dialogue systems is well-argued in [4], [5]. Each element of the semantic database is given a standard translation in French language. All in all, our semantic database contains 1,507 instances of actions, 1,121 instances of propositions and 2,086 instances of questions.

Automatic Pattern Generation: We considered three automatic pattern generation algorithms: a random mode and two DogmA-based modes. These modes produce interaction patterns as sequences of dialogue moves.

The random mode generates a pattern by randomly selecting for each move: a locutor (among two), a communicative function and a consistent semantic content. The communicative function is picked from 19 DIT++ general-purpose functions that occurred in interaction patterns of the study corpus. Pattern lengths are uniformly distributed between 2 and 5 .

The first DoGMA mode generates patterns based on a simple deliberative process that takes into account the information state and that exploits the semantic database. First, a dialogue game type and a compatible goal are chosen. Types are picked from a set that includes 3 actionoriented game types and 5 information-seeking-oriented game types. The probability to select a type corresponds to the distribution of types observed in the study corpus. Next, one of the DP is designated initiator of the game whereas the other is designated partner. The initiator plays an explicit proposition to establish the dialogue game. Then, the automatic generation starts. It takes advantage of the conventional behaviour manager of DoGMA to compute a time-ordered set of expected dialogue moves from the DPs. The algorithm that selects the next dialogue move takes into account: (i) the priority of an expected dialogue move (the more recent, the greater the priority is), and (ii) the interaction history (DPs cannot repeat an already produced dialogue move). The generation stops when no more dialogue moves are expected.

The second DoGMA mode simulates the implicit establishment of the game. It thus works similarly to the first one but only keeps the body of the pattern (entry and exit phases are discarded).

Natural Language Generation: Dialogue patterns are given a natural language representation through a simple template-based generation mechanism. For each dialogue move of a pattern, a template is selected and then applied. Generation templates have been manually crafted by extracting cue-phrases specific to communicative functions at the surface level of utterances of our study corpus. These templates obviously are language- and domain-dependent.

2) Database of $H-H$ Patterns: The database of $\mathrm{H}-\mathrm{H}$ interaction patterns was established by extracting all recurrent dialogue patterns from our reference corpus (process was similar to the one executed on the study corpus, cf. section III). Interaction patterns were post-processed on their surface level to obtain a normalised form. This process consisted in the removal of transcription signs (e.g., pause indicator), in the normalisation of punctuation and in the correction of spelling mistakes. In short, we extracted 44 $\mathrm{H}-\mathrm{H}$ dialogue patterns which mainly are adjacency pairs. An example of such a pattern from our corpus is: "- What I propose is to remove the subheading from the query. Alright!" (translated from French to English).

\section{B. Experiment}

Our experiment consists in the qualitative assessment of the interaction patterns obtained by the previously described means. Evaluation database contains 176 patterns (44 per mode). We considered two dimensions to carry out our comparative study: (i) coherence (a pattern is said to be coherent if it does not contain a dialogue contribution which presence is not easily explained by taking into account the previous ones [28]), and (ii) naturalness (a pattern is "natural" if it seems to have been produced by a $\mathrm{H}-\mathrm{H}$ interaction).

We have performed the evaluation on the web, in French. Participants were confronted to a random selection of patterns to avoid any effect on the results. For each pattern, we asked the participants to answer two questions (one per dimension) using a Likert scale of 4 points (e.g., "Coherent", "Rather coherent", "Rather incoherent", "Incoherent"). Participants were recruited via French mailing lists. 89 French- 


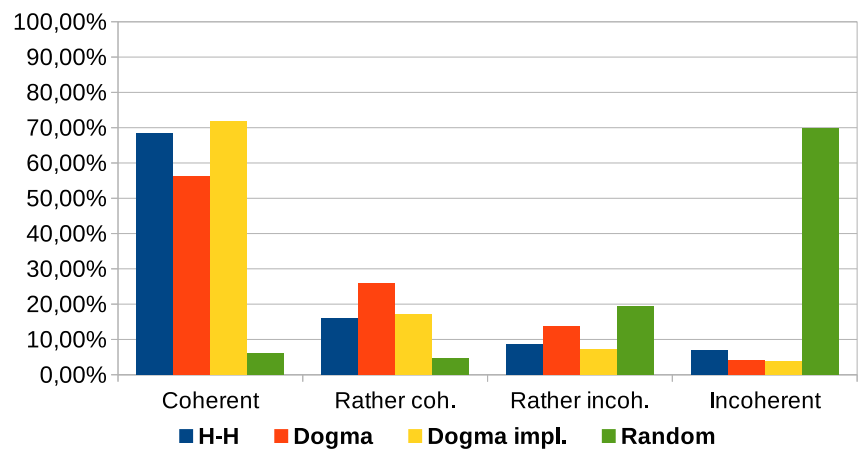

Figure 2. Distribution of coherence results per pattern groups. "DoGMA impl." stands for the second DoGMA-based generation mode.

native individuals have participated in this evaluation (26 females, 63 males). $75 \%$ of them are between 20 and 39 years old $(\min =19, \max =64)$.

\section{Results}

We collected 2,960 evaluations of pattern uniformly distributed over the 4 database types. Cumulative coherence results for each group are presented in figure 2. Two different clusters in terms of coherence can clearly be distinguished. The first one unites the set of $\mathrm{H}-\mathrm{H}$ patterns and the two DoGMA-generated sets of patterns. It is characterised by a common value of mode and median which is "Coherent", the highest level of coherence. The other cluster consists of the set of random-generated patterns (mode=median="Incoherent", the lowest level of coherence). Results for the random mode shows that humans are able to clearly assess coherence of short dialogue patterns.

We applied a statistical test to quantify a distance between the empirical coherence distribution of the H-H group and the other ones (Dogma, Dogma impl. and random). Due to the ordinal nature of our data and the large size of our samples, we selected the two-sample KolmogorovSmirnov test with a significance level $\alpha=0.05$. It turns out that the empirical coherence distributions of $\mathrm{H}-\mathrm{H}$ and "Dogma impl." groups can be said to come from the same distribution $(D=0.0459$, p-value $=0.412)$. However, this strong hypothesis is rejected for the Dogma group $\left(D=0.123\right.$, p-value $\left.=2.471 \times 10^{-05}\right)$ and for the random one $\left(D=0.736\right.$, $\mathrm{p}$-value $\left.=2.849 \times 10^{-173}\right)$. This test shows that there is no significant statistical difference between the observed empirical coherence distributions of the $\mathrm{H}-\mathrm{H}$ group and the "DoGMA impl." one. While being perceived coherent, patterns generated by the DoGMA mode seems to suffer from the explicit version of the game establishment process.

An analysis of the "naturalness" results reveals three different clusters. The first one consists of the set of $\mathrm{H}-\mathrm{H}$ patterns (mode=median="Human-like", the highest level). The second one is made of the sets of the two Dogma modes
(mode="Human-like", median="Rather human-like"). The third one contains the random mode which has been perceived "Non-human" (the lowest level). DogmA-generated patterns are distinguishable from $\mathrm{H}-\mathrm{H}$ patterns while being mainly seen as human-like. One obvious reason is that we use a simplistic template-based NLG system.

\section{Discussion}

We have outlined interesting properties of our dialogue game-based model. First, we have presented its ability to model in a computational way empirically observed $\mathrm{H}-\mathrm{H}$ dialogue patterns. Next, we have described how this model can be useful to manage the conventional communicative behaviour of an interacting agent, in particular by its discernment of legal moves from the current dialogue state. Notably, this model allows to manage dialogue in cases where a conventional response is sufficient, thus avoiding a complicated and difficult to set up plan-recognition process. Then, this framework includes mechanisms for the cocontrol of dialogue by both participant which could be useful in mixed-initiative interaction [2]. Last, we have shown that this model succeeds to produce a human level of coherence on both semantic and pragmatic levels on short dialogue patterns, contrary to the weak coherence reached by [10]. Our model thus seems very promising to manage the lowlevel communicative behaviour of an interactive agent while being integrable to a high-level deliberative process (e.g., through dialogue plans [30], activation networks [1]).

\section{CONCLUSions AND Future Work}

In this work, we have seen how dialogue patterns can be fruitfully used to enrich high-level structures for dialogue management, namely, dialogue games seen as structures capturing commitments. These games are building blocks of conventional interactions that an agent could employ to interact with a human. We have pointed out computational advantages of these structures on both interpretative and generative levels of dialogue management. We presented DogmA, an open-source module that can be used by an interactive agent to manage its conventional communicative behaviour in a two-interlocutor dialogue. We have shown that our library of empirically specified dialogue games can be used into DoGMA to generate fragments of dialogue that are coherent from a human perspective at the semantic and pragmatic levels.

Future work includes two interesting perspectives. One is the study of the implicitation of the dialogue game establishment process, that is not perceived as being "natural" in its explicit form. Another is the design of a high-level deliberative model that takes into account dialogue games and the opportunistic nature of dialogue. 


\section{REFERENCES}

[1] J. Cassell and T. Bickmore, "Negotiated collusion: Modeling social language and its relationship effects in intelligent agents," User Modeling and User-Adapted Interaction, vol. 13, no. 1-2, pp. 89-132, 2003.

[2] G. Tecuci, M. Boicu, and M. T. Cox, "Seven aspects of mixedinitiative reasoning: An introduction to this special issue on mixed-initiative assistants," AI Magazine, vol. 28, no. 2, p. 11, 2007.

[3] J. Mariani, S. Rosset, M. Garnier-Rizet, and L. Devillers, Natural Interaction with Robots, Knowbots and Smartphones: Putting Spoken Dialog Systems Into Practice. Springer Science \& Business Media, 2014.

[4] K. Jokinen and M. McTear, Spoken Dialogue Systems. Morgan \& Claypool, 2010, vol. 5.

[5] F. Landragin, Man-Machine Dialogue. Design and Challenges. London, UK: Wiley-ISTE, 2013.

[6] W. R. Swartout, J. Gratch, R. W. H. Jr., E. H. Hovy, S. Marsella, J. Rickel, and D. R. Traum, "Toward virtual humans," AI Magazine, vol. 27, no. 2, pp. 96-108, 2006.

[7] H. Clark, Using language. Cambridge University Press, 1996, vol. 4.

[8] J. Allwood, "Obligations and options in dialogue," Think Quarterly, vol. 3, pp. 9-18, 1994.

[9] J. Hulstijn, "Dialogue games are recipes for joint action," in Workshop on the Semantics and Pragmatics of Dialogue, 2000 .

[10] J. Orkin and D. Roy, "Automatic learning and generation of social behavior from collective human gameplay," in AAMAS, vol. 1, Richland, SC, 2009, pp. 385-392.

[11] G. Dubuisson Duplessis, N. Chaignaud, J.-P. Kotowicz, A. Pauchet, and J.-P. Pecuchet, "Empirical specification of dialogue games for an interactive agent," in PAAMS, 2013, pp. 49-60.

[12] S. Darmoni, J. Leroy, F. Baudic, M. Douyere, J. Piot, and B. Thirion, "Cismef : a structured health resource guide," Methods of Information in Medicine, vol. 39, pp. 30-35, 2000.

[13] W. Mann, "Dialogue games: Conventions of human interaction," Argumentation, no. 4, pp. 511-532, 1988.

[14] B. Grosz and C. Sidner, "Attention, intentions, and the structure of discourse," Computational Linguistics, vol. 12, no. 3, pp. 175-204, 1986.

[15] J. Allen, G. Ferguson, B. Miller, E. Ringger, and T. SikorskiZollo, "Dialogue systems: From theory to practice in TRAINS-96," in Handbook of Natural Language Processing, 2000, pp. 347-376.

[16] C. Rich and C. Sidner, "COLLAGEN: a collaboration manager for software interface agents," User Modeling and UserAdapted Interaction, vol. 8, no. 3, pp. 315-350, 1998.
[17] C. Rich and C. L. Sidner, "Using collaborative discourse theory to partially automate dialogue tree authoring," in Intelligent Virtual Agents, 2012, pp. 327-340.

[18] L. Polanyi and R. Scha, "A syntactic approach to discourse semantics," in International Conference on Computational Linguistics, 1984, pp. 413-419.

[19] N. Maudet, "Modéliser les conventions des interactions langagières: la contribution des jeux de dialogue," Ph.D. dissertation, Toulouse 3, 2001.

[20] T. Yuan, D. Moore, C. Reed, A. Ravenscroft, and N. Maudet, "Informal logic dialogue games in human-computer dialogue," Knowledge Engineering Review, vol. 26, no. 2, pp. 159-174, 2011.

[21] I. Lewin, "A formal model of conversational game theory," in Workshop on the Semantics \& Pragmantics of Dialogue, 2000 .

[22] M. Morge, F. Delecroix, and J.-C. Routier, "A virtual selling agent which is persuasive and adaptive," in Agreement Technologies, ser. Law, Governance and Technology Series, S. Ossowski, Ed. Springer Netherlands, 2013, vol. 8, pp. 625-645.

[23] H. Bunt, "The DIT++ taxonomy for functional dialogue markup," in AAMAS Workshop, Towards a Standard Markup Language for Embodied Dialogue Acts, 2009, pp. 13-24.

[24] E. A. Schegloff and H. Sacks, "Opening up closings," Semiotica, vol. 8, no. 4, pp. 289-327, 1973.

[25] S. Larsson and D. Traum, "Information state and dialogue management in the TRINDI dialogue move engine toolkit," Natural language engineering, vol. 6, no. 3\&4, pp. 323-340, 2000.

[26] D. N. Walton and E. Krabbe, Commitment in dialogue: Basic concepts of interpersonal reasoning. NY: State University of New York Press, 1995

[27] M. P. Singh, "Social and psychological commitments in multiagent systems," in AAAI Fall Symposium on Knowledge and Action at Social and Organizational Levels, 1991, pp. 104-106.

[28] W. Mann, "Dialogue macrogame theory," in SIGdial, 2002, pp. 129-141.

[29] T. Yuan, D. Moore, and A. Grierson, "A human-computer dialogue system for educational debate: A computational dialectics approach," International Journal of Artificial Intelligence in Education, vol. 18, no. 1, pp. 3-26, 2008.

[30] S. Larsson, "Issue-based dialogue management," Ph.D. Dissertation, Department of Linguistics, Goteborg University, Goteborg, 2002. 